\title{
Lymphoepithelioma-like gastric carcinoma located in the lesser curvature of the gastric body: A case report and review of the literature
}

\author{
QIUPING XU1 ${ }^{1}$, JUAN DU ${ }^{2}$ and BAORUI LIU ${ }^{3}$ \\ ${ }^{1}$ Nanjing Drum Tower Hospital, Clinical College of Traditional Chinese and Western Medicine, Nanjing University \\ of Chinese Medicine; ${ }^{2}$ Nanjing Drum Tower Hospital, Clinical College of Nanjing Medical University; \\ ${ }^{3}$ The Comprehensive Cancer Centre of Drum Tower Hospital, Medical School of Nanjing University \\ \& Clinical Cancer Institute of Nanjing University, Nanjing, Jiangsu 210008, P.R. China
}

Received September 24, 2015; Accepted December 8, 2015

DOI: $10.3892 / \operatorname{mco} .2015 .717$

\begin{abstract}
Lymphoepithelioma-like gastric carcinoma (LELC) is a type of Epstein-Barr virus (EBV)-associated gastric cancer, characterized by the presence of a lymphoid stroma with cells arranged primarily in microalveolar, thin trabecular and primitive tubular patterns, or isolated cells. In situ hybridization is usually used to confirm the presence of EBV. LELC is a rare type of cancer, with an incidence of 1-4\% among all gastric cancers. Despite its rarity, this tumor has attracted attention due to its distinct clinicopathological characteristics and its association with the immune system. This is the report of a rare case of gastric LELC located in the lesser curvature of the gastric body.
\end{abstract}

\section{Introduction}

Lymphoepithelioma-like gastric carcinoma (LELC) is a rare type of gastric cancer with distinct clinicopathological characteristics, which was first reported by Watanabe et al in 1976 (1). Over $80 \%$ of gastric LELCs have been proven to be associated with Epstein-Barr virus (EBV) infection (2,3). LELC, which has a male predominance and a favorable prognosis, is usually located in the upper and middle parts of the stomach. Computed tomography (CT) scan and endoscopic ultrasound (EUS) may assist in the diagnosis, which is confirmed by pathology. Since National Comprehensive Cancer Network (NCCN) guidelines have not been presented seperately for the treatment of LELC, it is usually treated as gastric adenocarcinoma. The aim of

Correspondence to: Dr Juan Du, Nanjing Drum Tower Hospital, Clinical College of Nanjing Medical University, No. 321 Zhongshan Road, Nanjing, Jiangsu 210008, P.R. China

E-mail: dujuanglyy@163.com

Key words: lymphoepithelioma-like carcinoma, Epstein-Barr virus, gastric cancer this report was to describe in detail this rare variant of gastric cancer and discuss its clinical characteristics and treatment.

\section{Case report}

A 41-year-old female patient presented with epigastric discomfort and general fatigue in April, 2014. A gastroscopy revealed a sizeable ulcer on the lesser curvature and posterior wall of the upper gastric body, with irregular borders, mucosal sclerosis and hemorrhagic tendency. Following biopsy and histological examination, the lesion was diagnosed as poorly differentiated gastric adenocarcinoma. The CT scan revealed thickening of the wall in part of the gastric body (Fig. 1). Based on these findings and considering the patient's age and general condition, a radical gastrectomy was performed with lymph node dissection and Roux-en-Y reconstruction.

The gross examination of the gastrectomy specimen revealed a tumor measuring $3 \times 2.5 \times 1 \mathrm{~cm}$ with a sizeable ulcer on the lesser curvature of the gastric body. The pathological examination revealed that the tumor consisted of nests of neoplastic cells within a dense lymphoid stromal infiltration (lymphoepitheloid carcinoma). Furthermore, the tumor invaded the muscular layer of the gastric wall, with nerve involvement, but without intravascular cancer emboli. The surgical margins were cancer-free and 2 of the 27 dissected lymph nodes at the lesser curvature were metastatic. The peritumoral gastric mucosa revealed mild chronic atrophic gastritis with intestinal metaplasia (Fig. 2).

On immunohistochemistry, the tumor cells were positive for human epidermal growth factor receptor 2 and E-cadherin, with a Ki-67 index of $60 \%$, but negative for CD133, epidermal growth factor receptor (EGFR), vascular EGFR2 and c-Met. In situ hybridization (ISH) confirmed Epstein-Barr encoding region (EBER) positivity (Fig. 3).

Finally, EBV-associated LELC of the stomach was diagnosed and staged as IIA (T2, N1, cM0) according to the NCCN guidelines, 2015 (http://www.nccn.org/professionals/drug_ compendium/content/changes_archive.asp?Panel_ID=40).

The patient received postoperative adjuvant chemotherapy [5-fluorouracil $1.8 \mathrm{~g} / \mathrm{m}^{2}$ as a $24-\mathrm{h}$ continuous intravenous 


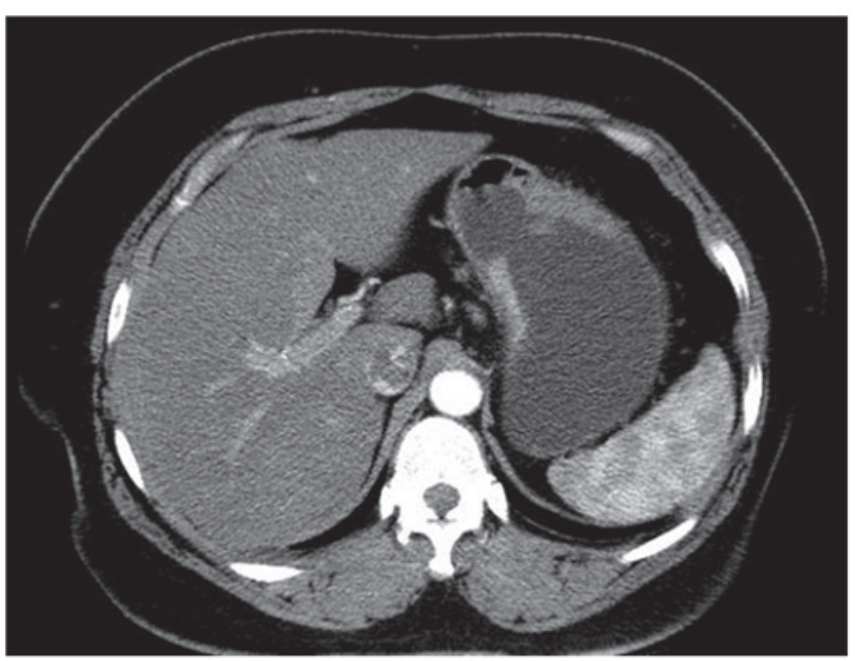

Figure 1. Contrast-enhanced computed tomography scan and coronal reformatted images showing thickening of the stomach wall in part of the gastric body.

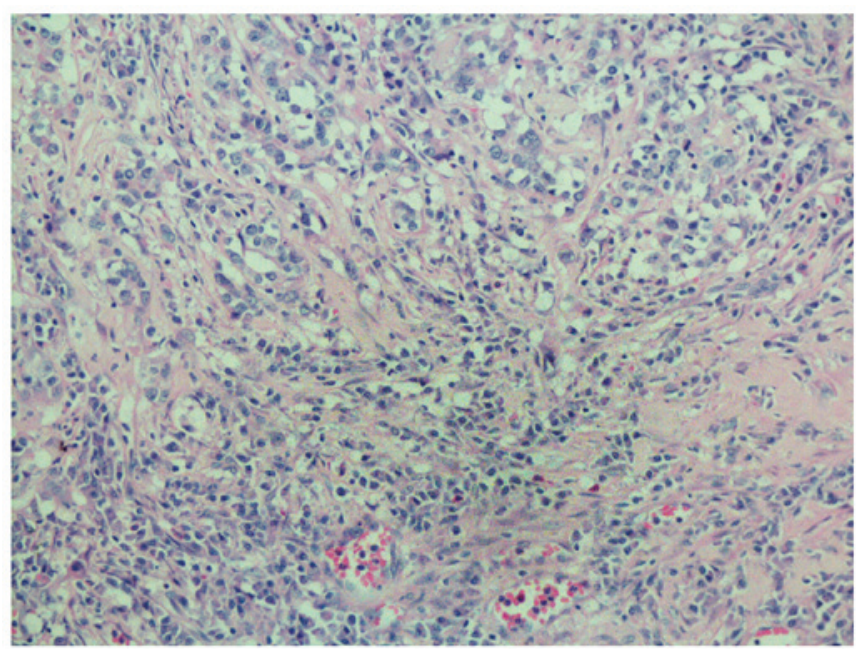

Figure 2. The tumor consisted of nests of neoplastic cells within a dense lymphoid stromal infiltration (hematoxylin and eosin stain; magnification, x200).

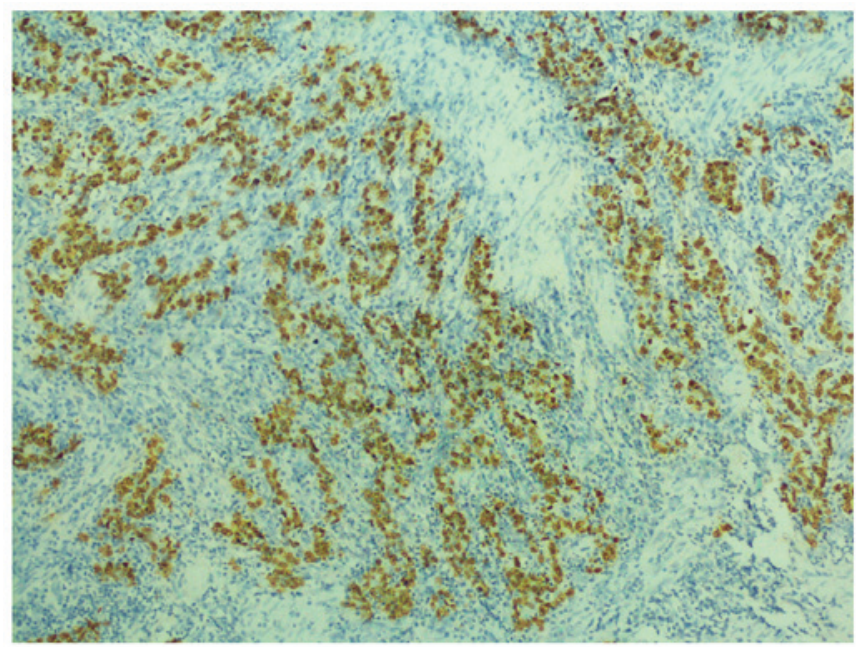

Figure 3. In situ hybridization confirmed Epstein-Barr encoding region (EBER) positivity. infusion + oxaliplatin $85 \mathrm{mg} / \mathrm{m}^{2}$ volume of distribution (VD) on day $1+$ leucovorin $100 \mathrm{mg} / \mathrm{m}^{2} \mathrm{VD}$ on day 1], without disease recurrence or metastasis during the 1 year follow-up after her initial diagnosis.

\section{Discussion}

EBV is a lymphotropic virus consisting of $184 \mathrm{kbp}$-sized double-stranded DNA, which belongs to the Herpesviridae family and infects $>90 \%$ of adults worldwide. EBV is closely associated with a wide range of human lymphoid and epithelial malignancies, including Burkitt's lymphoma, Hodgkin lymphoma, undifferentiated nasopharyngeal carcinoma (NPC) and EBV-associated gastric carcinoma (EBVaGC) (3). Furthermore, EBV has been isolated from various anatomic sites, including the salivary glands, thymus, larynx, lung, esophagus, uterine cervix, urinary bladder and skin (4).

EBVaGC is identified by the presence of EBV latent infection in neoplastic cells and its absence from normal epithelium or dysplastic lesions. Histopathologically, EBVaGC has two subtypes, namely LELC and ordinary adenocarcinoma (ordinary EBVaGC) (5). Gastric LELC consists of two subsets, namely EBV-positive and microsatellite instability (MSI)-high carcinomas $(6,7)$. As a rare form of gastric carcinoma, LELC was first reported as 'gastric cancer with a lymphoid stroma' by Watanabe et al in 1976, and was first demonstrated to be associated with EBV in a study published in 1990 (2).

The frequency of EBV infection in gastric carcinoma ranges from 2 to $20 \%$, with a worldwide mean frequency of $\sim 10 \%(8,9)$. However, due to geographical and environmental factors, the reported frequencies may differ. According to a meta-analysis published in 2009, the estimate of EBV positivity in gastric cancer is $8.7 \%$ (95\% confidence interval: $7.5-10.0 \%$ ) overall, and the frequencies are not statistically different among regions (9.9\% for American, 8.3\% for Asian and $9.2 \%$ for European cases). In addition, $15-25 \%$ of EBVaGCs exhibit the lymphoepithelioma-like pattern, whereas $86-91 \%$ of LELCs are EBV-positive $(10,11)$, suggesting that EBV infection, although not the sole cause, is closely associated with gastric LELC. The prevalence of MSI-high carcinoma in gastric LELC ranges from 7 to $39 \%$, with apparent geographic variability (6). Among the annual incidence of 951,600 gastric cancer cases worldwide (12), the patients who develop gastric LELC are estimated to be 9, 516-38,064/year.

LELC has distinct clinical characteristics that distinguish it from ordinary gastric adenocarcinoma. LELC exhibits a male predominance and is usually located in the proximal stomach. It was previousy reported that this type of cancer occurs in relatively younger patients, although the opposite observation has also been reported $(10,11)$. LELC often presents as an ulcerated or saucer-like tumor accompanied by marked thickening of the gastric wall. These characteristics may be well discernible on CT and EUS. In a CT study including $13 \mathrm{EBV}$-associated gastric carcinomas, the authors concluded that the carcinomas were generally located in the upper gastric region, with a large thickness-to-width ratio, or presented as a bulky mass projecting from the wall. However, gastric LELC had various CT appearances, including focal mucosal thickening, marked thickening of the wall with contrast enhancement, and bulky mass formation (13). It is 
difficult to distinguish LELCs presenting as a bulging mass from lymphomas, gastrointestinal stromal tumors, neurogenic tumors and glomus tumors by imaging alone. A comparison with the clinical findings of gastroscopy and pathology was recommended. EUS may easily detect the nodules of lymphoid stromal infiltration in the submucosa as a hypoechoic mass in the hyperechoic third layer (14). EBER-ISH has been used as a gold standard to identify EBV-associated gastric cancer $(3,15)$. The differential diagnosis of gastric LELC includes neuroendocrine tumors, malignant melanoma, intense lymphoid infiltrate alone, or even lymphoma.

In recent years, significant progress has been made in the multimodality treatment of gastric adenocarcinoma, particularly in terms of novel chemotherapeutic agents, novel targeted therapies and radiotherapy. However, the optimal treatment for gastric LELC remains unknown. Due to the rare occurrence of LELC, there are no specific NCCN guidelines for its treatment alone; thus, it is usually treated as gastric adenocarcinoma. Since the tumor boundaries are often well-defined and the majority of the patients are at a relatively early clinical stage, endoscopic submucosal dissection or surgical resection remains the mainstay of treatment. Nasopharyngeal LELC is highly sensitive to radiotherapy, which has become the main therapeutic approach. Although gastric LELC shares similar histological characteristics to undifferentiated NPC, its sensitivity to radiotherapy has not been reported. However, abdominal radiotherapy is difficult to target accurately and is associated with a relatively high complication rate.

Therapeutic approaches require further research on virus-host interactions in EBV-associated neoplasms (16). Since viral replication is inhibited by promoter methylation in EBV DNA, demethylating agents, such as 5-azacytidine, may induce lytic infection by EBV, leading to lysis of infected cells (17). The approach has particular merits in EBV-associated gastric cancer, in which methylation of the tumor suppressor gene is also a key abnormality. However, the efficiency of drug delivery and the side effects of demethylating agents require further investigation. Since most gastric LELCs contain the EBV oncoproteins, and cancer-driving viral antigens are highly attractive therapeutic targets, therapeutic vaccines targeting the EBV oncoproteins may prove to be beneficial. Furthermore, adoptive T-cell therapy is emerging as a promising cancer treatment, and one recently published study demonstrated that durable, complete regression of metastatic cervical cancer may occur after a single infusion of HPV-targeted tumor-infiltrating lymphocytes (TILs) (18). Hence, we must investigate whether an infusion of EBV-targeted TILs may induce cancer regression in patients with gastric LELC. Another novel finding, which is characteristic of EBVaGC, was recurrent amplification at 9p24.1 at the locus that includes Janus kinase 2, programmed death-ligand 1 (PD-L1) and PD-L2. Blocking the interaction between PD-1 and PD-L1/L2 may augment the antitumor immune response and clinical trials investigating the efficacy of immunotherapy by targeting these molecules are under way (19-21).

LELC has a relatively favorable prognosis. Tak et al (22) compared the clinical characteristics and prognostic factors between gastric LELC and gastric adenocarcinoma, and found that postoperative recurrence or metastasis tended to occur less frequently in gastric LELC compared with poorly-differentiated gastric adenocarcinoma. Among prognostic factors, only the number of lymph node metastases exhibited a significant difference, with gastric LELC being associated with a smaller number of lymph node metastases. The disease-free and overall survival rates of gastric LELC were higher compared with those of poorly-differentiated gastric adenocarcinoma. Park et al (23) demonstrated that age, histological type, Lauren classification, tumor location, depth of invasion, lymph node metastasis and venous invasion were independent prognostic factors, whereas the LELC type was not. The 5-year survival rate of the LELC group (97.7\%) was better compared with that of the non-LELC group (89.4\%). Kim et al (24) reached the same conclusion by investigating the clinicopathological characteristics and prognosis of LELC, signet ring cell, mucinous and papillary carcinomas in cases with advanced gastric cancer.

Gastric LELC is a distinct subtype of gastric carcinoma with regard to its clinical characteristics. LELC generally has a better prognosis compared with other types of EBV-associated gastric carcinomas and conventional gastric carcinomas. Pathologists and clinicians should take into consideration this subset of gastric cancer to make an accurate diagnosis and select the appropriate treatment.

\section{References}

1. Watanabe H, Enjoji M and Imai T: Gastric carcinoma with lymphoid stroma. Its morphologic characteristics and prognostic correlations. Cancer 38: 232-243, 1976.

2. Burke AP, Yen TS, Shekitka KM and Sobin LH: Lymphoepithelial carcinoma of the stomach with Epstein-Barr virus demonstrated by polymerase chain reaction. Mod Pathol 3: 377-380, 1990.

3. Shinozaki-Ushiku A, Kunita A and Fukayama M: Update on Epstein-Barr virus and gastric cancer (Review). Int J Oncol 46: 1421-1434, 2015.

4. Cheng N, Hui DY, Liu Y, Zhang NN, Jiang Y, Han J, Li HG, Ding YG, Du H, Chen JN and Shao CK: Is gastric lymphoepithelioma-like carcinoma a special subtype of EBV-associated gastric carcinoma? New insight based on clinicopathological features and EBV genome polymorphisms. Gastric Cancer 18: 246-255, 2015.

5. Fukayama $M$ and Ushiku T: Epstein-Barr virus-associated gastric carcinoma. Pathol Res Pract 207: 529-537, 2011.

6. Grogg KL, Lohse CM, Pankratz VS, Halling KC and Smyrk TC: Lymphocyte-rich gastric cancer: Associations with Epstein-Barr virus, microsatellite instability, histology, and survival. Mod Pathol 16: 641-651, 2003.

7. Bittar Z, Fend F and Quintanilla-Martinez L: Lymphoepithelioma-like carcinoma of the stomach: A case report and review of the literature. Diagn Pathol 8: 184, 2013.

8. Fukayama M: Epstein-Barr virus and gastric carcinoma. Jpn J Clin Med 70: 1715-1719, 2012 (In Japanese).

9. Chen JN, He D, Tang F and Shao CK: Epstein-Barr virus-associated gastric carcinoma: A newly defined entity. J Clin Gastroenterol 46: 262-271, 2012.

10. Murphy G, Pfeiffer R, Camargo MC and Rabkin CS: Meta-analysis shows that prevalence of Epstein-Barr virus-positive gastric cancer differs based on sex and anatomic location. Gastroenterology 137: 824-833, 2009.

11. Lee JH, Kim SH, Han SH, An JS, Lee ES and Kim YS: Clinicopathological and molecular characteristics of Epstein-Barr virus-associated gastric carcinoma: A meta-analysis. J Gastroenterol Hepatol 24: 354-365, 2009.

12. Torre LA, Bray F, Siegel RL, Ferlay J, Lortet-Tieulent J and Jemal A: Global cancer statistics, 2012. CA Cancer J Clin 65: 87-108, 2015.

13. Maeda E, Akahane M,Uozaki H, Kato N,Hayashi N, Fukayama M and Ohtomo K: CT appearance of Epstein-Barr virus-associated gastric carcinoma. Abdom Imaging 34: 618-625, 2009.

14. Bai Y, Gao Q, Ren G, Wang B and Xiang H: Epstein-Barr virus-associated lymphoepithelioma-like gastric carcinoma located on gastric high body: Two case reports. Indian J Pathol Microbiol 57: 463-466, 2014 
15. Uozaki $\mathrm{H}$ and Fukayama M: Epstein-Barr virus and gastric carcinoma - viral carcinogenesis through epigenetic mechanisms. Int J Clin Exp Pathol 1: 198-216, 2008.

16. Fu DX, Tanhehco Y, Chen J, Foss CA, Fox JJ, Chong JM, Hobbs RF, Fukayama M, Sgouros G, Kowalski J, et al: Bortezomib-induced enzyme-targeted radiation therapy in herpesvirus-associated tumors. Nat Med 14: 1118-1122, 2008.

17. Jung EJ, Lee YM, Lee BL, Chang MS and Kim WH: Lytic induction and apoptosis of Epstein-Barr virus-associated gastric cancer cell line with epigenetic modifiers and ganciclovir. Cancer Lett 247: 77-83, 2007.

18. Stevanović S, Draper LM, Langhan MM, Campbell TE, Kwong ML, Wunderlich JR, Dudley ME, Yang JC, Sherry RM, Kammula US, et al: Complete regression of metastatic cervical cancer after treatment with human papillomavirus-targeted tumor-infiltrating T cells. J Clin Oncol 33: 1543-1550, 2015.

19. Dolan DE and Gupta S: PD-1 pathway inhibitors: Changing the landscape of cancer immunotherapy. Cancer Control 21: 231-237, 2014.

20. Naidoo J, Page DB and Wolchok JD: Immune modulation for cancer therapy. Br J Cancer 111: 2214-2219, 2014.
21. Kim SY, Park C, Kim HJ, Park J, Hwang J, Kim JI, Choi MG, Kim S, Kim KM and Kang MS: Deregulation of immune response genes in patients with Epstein-Barr virus-associated gastric cancer and outcomes. Gastroenterology 148: 137-147.e9, 2015.

22. Tak DH, Jeong HY, Seong JK, Moon HS and Kang SH: Comparison of clinical characteristics and prognostic factors between gastric lymphoepithelioma-like carcinoma and gastric adenocarcinoma. Korean J Gastroenterol 62: 272-277, 2013 (In Korean).

23. Park S, Choi MG, Kim KM, Kim HS, Jung SH, Lee JH, Noh JH, Sohn TS, Bae JM and Kim S: Lymphoepithelioma-like carcinoma: A distinct type of gastric cancer. J Surg Res 194: 458-463, 2015.

24. Kim KH, Kim MC, Jung GJ and Kim SJ: The differences in clinicopathological features and prognosis among the subtypes of signet ring cell, mucinous, papillary, and lymphoepithelioma-like carcinoma in advanced gastric cancer. Hepatogastroenterology 61: 2149-2155, 2014. 